\title{
A Study of the Dynamics of the Heme Pocket and C-Helix in CooA Upon CO Dissociation Using Time-Resolved Visible and UV Resonance Raman Spectroscopy
}

\author{
Akihiro Otomo, ${ }^{\dagger}$ Haruto Ishikawa, ${ }^{\dagger}$ Misao Mizuno, ${ }^{\dagger}$ Tetsunari Kimura,,$\stackrel{\ddagger}{ }$ Minoru Kubo, ${ }^{\ddagger}$ \\ Yoshitsugu Shiro, ${ }^{\ddagger}$ Shigetoshi Aono,${ }^{\S}$ and Yasuhisa Mizutani ${ }^{*} \dagger$ \\ 'Department of Chemistry, Graduate School of Science, Osaka University, 1-1 Machikaneyama, Toyonaka, Osaka \\ 560-0043, Japan, "RIKEN SPring-8 Center, 1-1-1 Kouto, Sayo, Hyogo 679-5148, Japan and ${ }^{\S}$ Okazaki Institute for \\ Integrative Bioscience, National Institutes of Natural Sciences, 5-1 Higashiyama, Myodaiji, Okazaki 444-8786, Japan, \\ \#Present address; Department of Chemistry, Graduate School of Science, Kobe University, 1-1 Rokkodai, Kobe, \\ Hyogo 657-8501, Japan
}

Data Analysis for the Time-resolved Resonance Raman Measurement of CO-ligated form of CooA. Figure S1 shows the high-frequency region of the visible Raman spectra of CooA. Trace (a) is the observed time-resolved resonance Raman spectrum of CooA at negative time delay that represents the spectral feature of the sample before photodissociation by the pump pulse. The $v_{4}$ band of the time-resolved resonance Raman spectrum for CO-ligated form of CooA at $-200 \mathrm{~ns}$ has distinguishable positions at $1371 \mathrm{~cm}^{-1}$ and $1359 \mathrm{~cm}^{-1}$. In our previous timeresolved resonance Raman studies on hemeproteins, heme-ligand religation was completed in photolyzed samples at negative time delay. However, the photodissociated form, Pro2-ligated form of CooA, was accumulated which may occur during the time-resolved resonance Raman measurement of the CO-ligated form of CooA. To obtain the spectral contribution of the photodissociated forms of CooA, we calculated difference spectra by subtracting the spectrum of the CO-ligated form of CooA (trace (b)) from the time-resolved resonance Raman spectra (trace (a)). The difference spectrum (trace (c)) was identical to the spectrum of the Pro2-ligated form of CooA (trace (d)). Therefore, we concluded that the Pro2-ligated form of CooA was accumulated during the measurements. Since the observed time-resolved visible resonance Raman spectra of CooA included contributions from CO-ligated, photodissociated, and the accumulated Pro2ligated forms of CooA, we calculated difference spectra by subtracting both the spectrum of the $\mathrm{CO}$-ligated and the accumulated Pro2-ligated forms from the observed time-resolved visible resonance Raman spectra. The factor of subtraction was determined based on the intensities of the $v_{4}$ bands of the CO-ligated and the accumulated Pro2-ligated forms. 


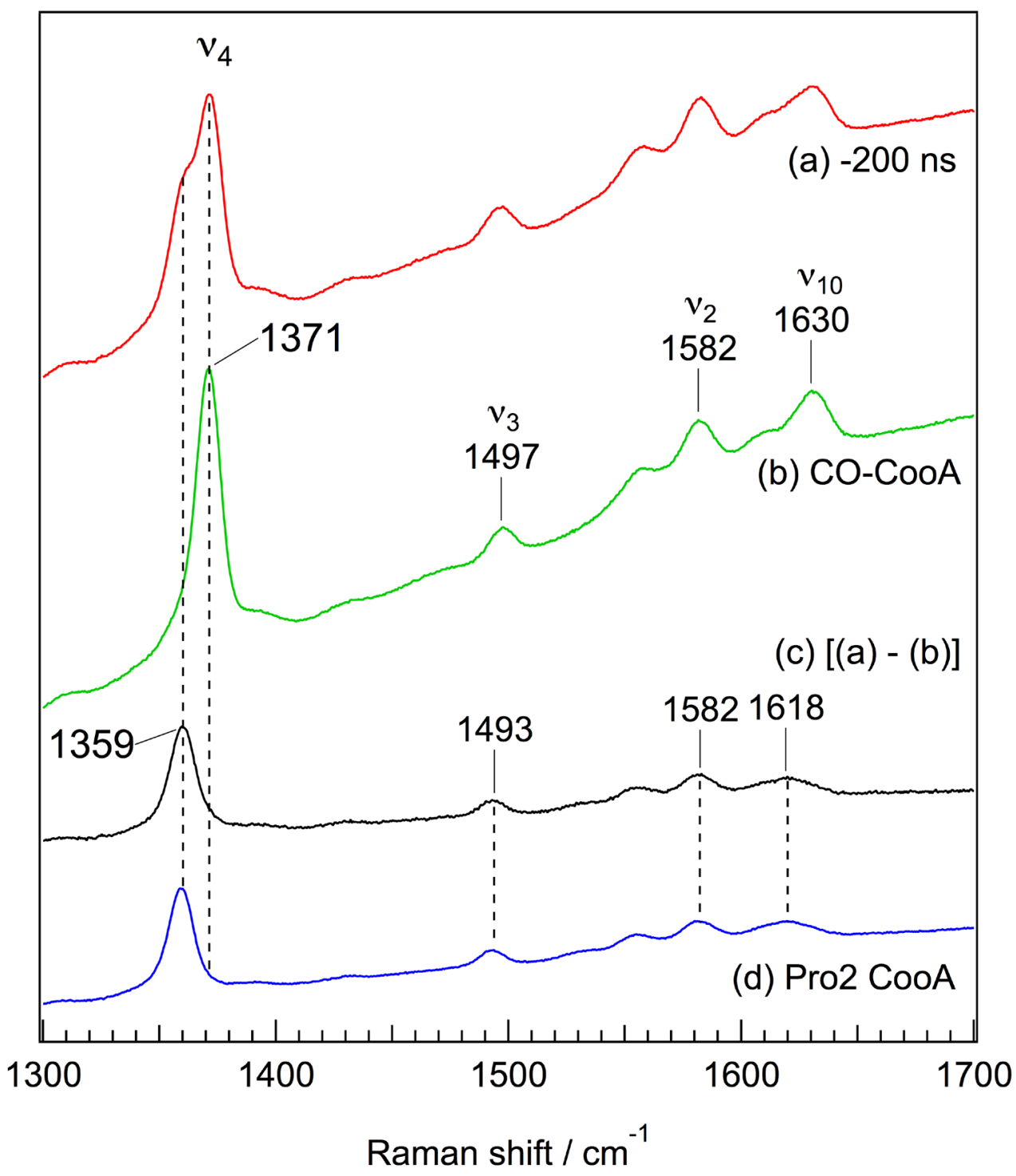

Figure S1. Visible resonance Raman spectra of CooA. Trace (a): nanosecond time-resolved visible resonance Raman spectrum of the $\mathrm{CO}$-ligated form of CooA upon $\mathrm{CO}$ photodissociation for $-200 \mathrm{~ns}$ probe pulse delay times with respect to pump pulse in the $1300-1700 \mathrm{~cm}^{-1}$ region. Trace (b): the static visible resonance Raman spectrum of CO-ligated form of CooA. Trace (c): the difference spectrum by subtracting the spectrum of the CO-CooA form (trace (b)) from the time-resolved resonance Raman spectra (trace (a)). Trace (d): the static visible resonance Raman spectrum of Pro2-ligated form of CooA. 
Transient Absorption Measurement of CooA. The transient absorption measurement was operated at $10 \mathrm{~Hz}$ and $20 \mathrm{~Hz}$ for the pump and the probe pulses, respectively. A nanosecond $\mathrm{Nd}$ :YAG laser (Continuum, Minilite I) was used for the $532 \mathrm{~nm}$ pump pulse for the photodissociation of CO and a xenon flash lamp (Hamamatsu Photonics, L2360) was utilized for the probe pulse with the time resolution of $\sim 2 \mu \mathrm{s}$. Absorption spectra were collected using a fiber-coupled spectrometer (Ocean Optics, USB2000+), which was managed using LabVIEW2012 software (National Instruments) on a personal computer. To avoid the accumulation of the Pro2-ligated form of CooA during the measurement, we employed a continuous flow system. The sample cell consisted of $\mathrm{CaF}_{2}$ and quartz windows separated by a 1 mm stainless steel sheet. The sample concentration was $40 \mu \mathrm{M}$ with a flow rate $500 \mu \mathrm{L} / \mathrm{min}$.

Figure S2 (A) shows the transient absorption spectra at 0 to $1000 \mu$ s delay times for CO-ligated form of CooA. The negative absorption band centered at $421 \mathrm{~nm}$ represents the photodissociation of $\mathrm{CO}$ from $\mathrm{CO}$-ligated form of CooA. The intensity changes of the negative absorption band are due to the rebinding of $\mathrm{CO}$ to CooA. Since the positive absorption band resulted from the photodissociated species, the transient species were derived from Pro2-ligated form and 5coordinate form of CooA. The positive absorption band position at $438 \mathrm{~nm}$ was different from the static difference spectrum of CO-ligated and Pro2-ligated form of CooA (Figure S2 (B)), which indicating 5-coordinate form of CooA. In the transient absorption spectra, the positive absorption band exhibited intensity decrease and slightly blue-shift at later delay time. To examine the spectral changes at later delay time, we calculated difference spectrum between the normalized transient absorption spectra at $0 \mu \mathrm{s}$ and $200 \mu \mathrm{s}$ (Figure S2 (C)). The small positive peak at $431 \mathrm{~nm}$ indicates the blue-shift at $200 \mu \mathrm{s}$. The blue-shift indicates a formation of Pro2ligated form of CooA, while the intensity decrease comes from the $\mathrm{CO}$ rebinding to Pro2-ligated form or 5-coordinate form of CooA. Figure S2 (D) shows the transient absorption spectra at millisecond delay times for CO-ligated form of CooA. The difference spectrum between $20 \mathrm{~ms}$ and $980 \mathrm{~ms}$ (red line) was very similar to that for the static difference spectrum of CO-ligated and Pro2-ligated form of CooA (Figure S2 (B)), indicating that the Pro2-ligated form of CooA is the only photoproduct at $20 \mathrm{~ms}$. The Pro-2 ligated form could be accumulated in the sample for the time-resolved resonance Raman measurements.
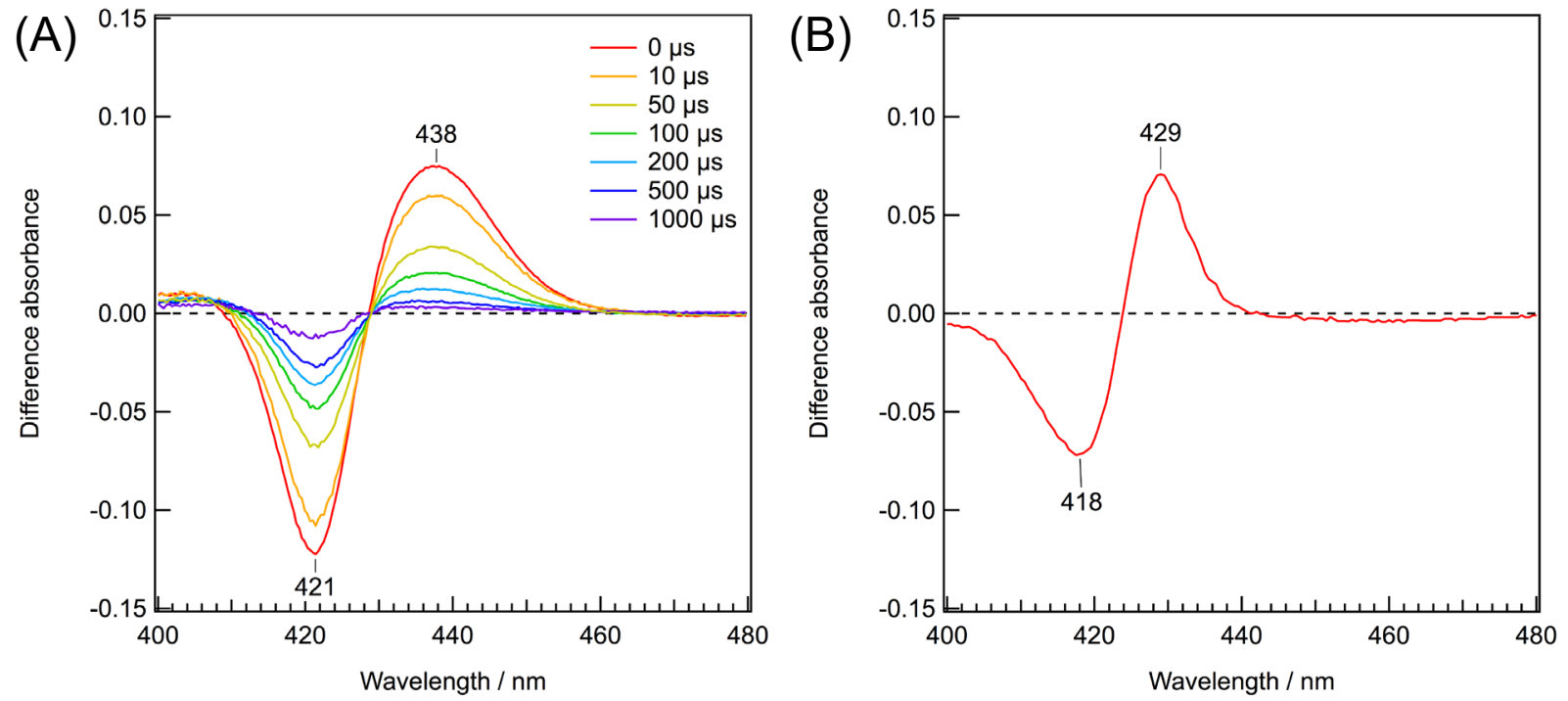

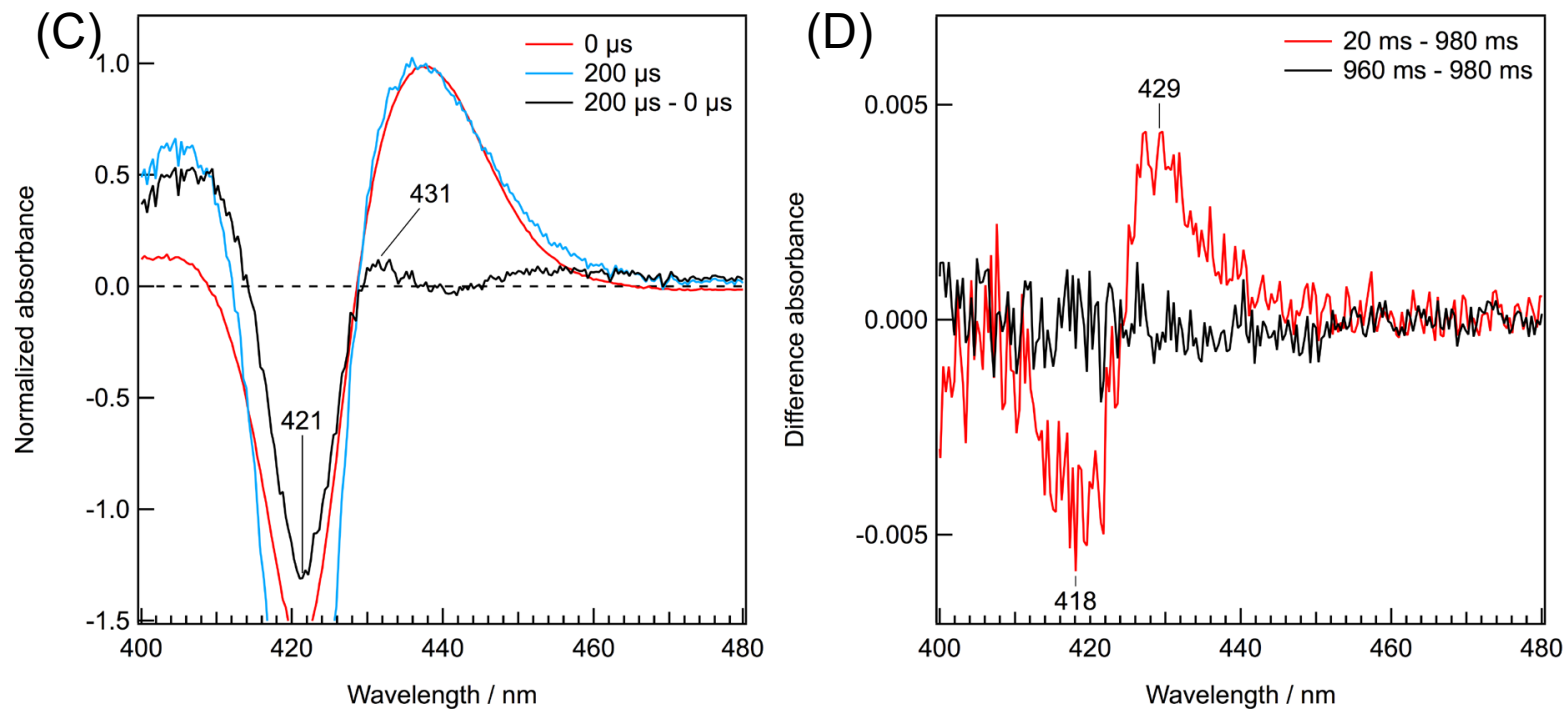

Figure S2. Transient absorption spectra of CO-ligated form of CooA after the photodissociation of CO. (A) Transient absorption spectra of CO-ligated form of CooA from $0 \mu$ s to $1000 \mu$ s in the 400-480 nm region. (B) Static difference spectrum of CO-ligated form and Pro2-ligated form of CooA in the 400-480 nm region. (C) Normalized transient absorption spectra of CO-ligated form of CooA after the photodissociation of $\mathrm{CO}$ at $0 \mu \mathrm{s}$ and $200 \mu \mathrm{s}$. Black line indicates the difference spectrum of the normalized transient absorption spectra at $0 \mu \mathrm{s}$ and $200 \mu \mathrm{s}$. (D) Transient absorption spectra of CO-ligated form of CooA after the photodissociation of $\mathrm{CO}$ at millisecond time scale. Red line represents the difference spectrum between $20 \mathrm{~ms}$ and $980 \mathrm{~ms}$. Black line represents the difference spectrum between $960 \mathrm{~ms}$ and $980 \mathrm{~ms}$. 